\title{
The Variable Rate Intravenous Insulin Infusion in Clinical Practice 2015: an audit against the new JBDS guidance
}

\author{
HYMA RACHABATTULA, ${ }^{1}$ EMILY SIMMONS, ${ }^{1}$ SONYA GHATAHORA, ${ }^{1}$ PAUL GRANT1,2
}

\begin{abstract}
Background: Variable rate intravenous insulin infusion (VRIII) is an established method to achieve normoglycaemia in unwell or fasting patients. The Joint British Diabetes Societies (JBDS) released new guidance for the use of VRIII in medical inpatients in October 2014. This specifies the appropriate clinical circumstances in which a VRIII should be used and how it should be initiated, managed and discontinued safely.

Methods: We developed an audit tool based on the guidelines and audited the current practice at the Royal Sussex County Hospital, Brighton, prior to the roll out of the new standards. We have audited $\mathbf{5 0}$ patients on VRIII under non-specialist medical and surgical care.

Results: Several parameters were checked. VRIII prescription was signed appropriately as per the guidance in $98 \%$ of patients. Oral hypoglycaemic medications were omitted in $83 \%$. Short-acting and mix insulins were omitted only in $88 \%$. Long-acting insulin was administered only in $77 \%$ of the patients. Blood glucose was tested 1-2 hourly in $90 \%$ of patients. All patients with hypoglycaemic episodes were treated as per protocol. On the occasions where VRIII was discontinued, it was reinitiated within $\mathbf{2 0}$ min as per the guidelines only in $36 \%$ of cases. In patients with persistent hyperglycaemia, the rate was increased in $80 \%$ of cases.
\end{abstract}

Discussion: Clinical practice surrounding the use, appropriate management and monitoring parameters appeared to fall short of the standards suggested by the latest JBDS guidance. Our audit outcome was to prepare a protocol for the trust summarising the indications for VRIII, target capillary blood glucose, how and when to stop VRIII for patients with diabetes and also a prompt to prescribe basal insulin for patients with diabetes and when a referral should be made to the diabetes inpatient team. Intro-

Department of Diabetes, Royal Sussex County Hospital, Eastern Road, Brighton.

Oxford Centre for Diabetes, Endocrinology and Metabolism, Churchill Hospital, Oxford.

Address for correspondence: Paul Grant

E-mail: drpaul.grant@doctors.org.uk

http://dx.doi.org/10.15277/bjd.2016.059 ducing teaching sessions to all the professionals and raising the awareness through a hospital communication programme on the wards would also help to theoretically improve the results.

Conclusions: The majority of patients on VRIII are managed by non-diabetic teams and practice may vary from set guidance due to unfamiliarity and lack of diabetes specific knowledge. The appropriate use of VRIII needs to be tackled through remedial education, introduction of the new JBDS guidance and allied to improvements in systems and processes.

Br J Diabetes 2016;16:25-29

Key words: insulin sliding scale, VRIII, JBDS, insulin safety

\section{Introduction}

Approximately $15 \%$ of hospital inpatients have diabetes, of which $70 \%$ are admitted as medical emergencies; the majority have diabetes as a secondary diagnosis. ${ }^{1}$ There is good evidence from both medical and surgical settings to indicate that, if blood glucose is not controlled, the outcomes measured by mortality, morbidity or length of stay are suboptimal. Variable rate intravenous insulin infusion (VRIII) or sliding scales have been used for many years as an effective mechanism of adjusting and controlling blood glucose levels in the hospital setting. It is well recognised that there is no such thing as one perfect sliding scale, ${ }^{2}$ but it does provide a good system of stabilising a patient's diabetes following the inter-current stresses of medical illness, surgery and metabolic imbalance. ${ }^{3,4}$

Results from the National Diabetes Inpatient Audit over recent years have demonstrated that there is a great deal of variation in practice around the use of VRIII in terms of the appropriateness of its use, the individualised settings, its duration and effectiveness and the recurrent problem of general nursing and medical staff's reluctance to continue the administration of basal insulin alongside the insulin infusion. ${ }^{5}$ The previous National Diabetes Inpatients Audit results from 2013-14 demonstrated 5 :

1 Inappropriate VRIII use: $6.5 \%$ of patients were thought to have been treated with a VRIII unnecessarily.

2 Inappropriate duration of use: $10.6 \%$ of inpatients with diabetes received an insulin infusion during the previous 7 days, of whom $7.8 \%$ were treated with an insulin infusion for 7 days or longer; $7.7 \%$ of insulin infusions were deemed unnecessarily long by the diabetes team. 
Figure 1. Audit tool table

\begin{tabular}{|l|l|l|}
\hline Patient details & Treat hypo as per protocol & \\
\hline Ward-med/surg & Reinitiate VRIII within 20 mins & \\
\hline Gender & If persistent hypos, reduce the rate & \\
\hline Age & If persistent hyper >12, increase the rate & \\
\hline Known type 1 DM & Document of fluid status & \\
\hline Known type 2 DM & U\&E check daily & \\
\hline If T2, on insulin & Assess the need to continue on VRIII & \\
\hline New diagnosis of T1DM & Duration of VRIII, approximate no. of hrs & \\
\hline New diagnosis of T2DM & On VRIII >7 days & \\
\hline Reason for admission & Wearning off VRIII & \\
\hline Indication for VRIII & Eating and drinking & \\
\hline Prescription A signed & Stable CBG, 6-10(4-12) & \\
\hline Oral hypos omitted & Changed at mealtime & \\
\hline Short-acting/mix insulin omitted & Basal was given Yes/No \\
\hline Long-acting insulin administered & Restarted oral hypos \\
\hline Blood glucose test frequency & Check CBGS an hour after stopping VRIII & \\
\hline
\end{tabular}

3 Inadequate monitoring: 1.7\% of patients on an insulin infusion had between one and three glucose measurements in the previous $24 \mathrm{~h}$ (equivalent to less than one every $8 \mathrm{~h}$ ), with $0.8 \%$ having no glucose monitoring in the previous $24 \mathrm{~h}$.

In October 2014 the Joint British Diabetes Societies (JBDS) produced welcome new guidance on the use of VRIII in order to provide clarity and evidence for effective use in a clinical context. ${ }^{6}$ It was designed to "cover adult in-patients with medical conditions and diabetes/hyperglycaemia who require IV administration of insulin to keep their blood glucose within the recommended target range during an acute illness or a period of starvation". Heterogeneity between trusts has traditionally led to "increased risk of errors which can potentially lead to significant morbidity and mortality. It also makes it inherently difficult to study its efficacy, optimisation and safety profile. In addition, despite guidelines, both local and national audits have shown that VRIII is often used when not indicated, its duration is unnecessarily prolonged and the step down to other glucose lowering medication is often not practiced safely".

"It is hoped that its adoption nationally will help harmonise the use of the VRIII and therefore enable multicentre studies to be carried out in order to allow continual refinement in its use".

We wished to focus on the processes that are followed with regard to the use of VRIII. This present audit therefore provides a snapshot of current practice within our trust to look at our baseline level of compliance with the JBDS standards prior to them being rolled out. This was in order to identify where the deficits lay and to help inform service improvement. It also provides the opportunity to critique the guidance when applied to day-to-day clinical activity.

\section{Methods}

A retrospective observational approach was used to gather data on the use of VRIII within our hospital trust. Local audit registration and governance procedures were followed. Data collection took place over a 12-week period. Adult patients under non-
Table 1 Reasons for use of variable rate intravenous insulin infusion (VRIII) in this snapshot audit

\begin{tabular}{ll}
\hline Indication for VRIII & Number (\%) \\
Nil by mouth/poor oral intake & $22(44 \%)$ \\
Hyperglycaemia & $15(30 \%)$ \\
Diabetic ketoacidosis & $9(18 \%)$ \\
HHS & $2(4 \%)$ \\
Sepsis & $2(4 \%)$
\end{tabular}

specialist care were included and there were no obvious exclusion criteria as we wanted to assess the clinical management of as many patients as possible. We developed an audit tool (Figure 1) based on these guidelines and audited the current practice at the Royal Sussex County Hospital, Brighton.

\section{Results}

Fifty patients in the inpatient setting who were on VRIII for a variety of reasons were audited. Their mean age was 62 years; $80 \%$ were male and $20 \%$ were female. Of these patients, $4 \%$ had a new diagnosis of type 1 diabetes and $42 \%$ were already known to have type 1 diabetes. Similarly, $4 \%$ of the patients had a new diagnosis of type 2 diabetes and $56 \%$ of patients were known to have type 2 diabetes, $32 \%$ of whom were treated with insulin. Indications for the use of VRIII are listed in table 1.

\section{Appropriate set-up}

VRIII prescription was signed appropriately as per the guidance in $98 \%$ of patients. Oral hypoglycaemic medications were omitted in $83 \%$. Short-acting and mix insulins were omitted in $88 \%$ of patients. Basal/long-acting insulin was administered only in $77 \%$ of the patients. Capillary blood glucose was tested 1-2 hourly in $90 \%$ of patients whilst on VRIII.

\section{Management of dysglycaemia}

All patients with hypoglycaemic episodes were treated as per the trust protocol. On the occasions where VRIII was discontinued, it was reinitiated within 20 min as per the guidelines in only $36 \%$ of cases (this is deemed necessary in order to prevent rebound hyperglycaemia). Two patients had persistent hypoglycaemia; the insulin rate was reduced only in one case. In patients with persistent hyperglycaemia, the rate was appropriately increased in $80 \%$ of cases.

\section{Fluid and electrolyte status}

Fluid status was noted and fluid balance charts were documented in only $52 \%$ of patients. $78 \%$ of patients had daily renal function and electrolyte check ( $92 \%$ of medical and $63 \%$ of surgical patients). In two-thirds of patients (66\%) the need to continue the VRIII was appropriately re-assessed and documented in the medical records. None of the patients had VRIII for more than 7 days in total.

\section{Discontinuing VRIII}

$80 \%$ of patients were weaned off VRIII when eating and drinking. Two patients were on nasogastric feeds. $82 \%$ of patients had stable 
capillary blood glucose (CBG) when switched to subcutaneous insulin. $85 \%$ of them were weaned off at a meal time as per the best practice guidance. Basal insulin was given prior to switching off VRIII in $84 \%$ of patients on average. Short-acting insulin and oral hypoglycaemic medications were restarted prior to switching from VRIII in $52 \%$ of cases. CBG was monitored $1 \mathrm{~h}$ after stopping VRIII (which is also part of the protocol) in only $58 \%$ of patients.

In summary, the major deviations from meeting the JBDS standards related to issues with appropriate use and duration of VRIII; failure to continue basal insulin; failure to restart a sliding scale after hypoglycaemia; suboptimal monitoring and documentation of fluid status and renal function; insufficient clarity and documentation over the continuance of VRIII; and poor practice around the weaning/discontinuation of VRIII.

\section{Discussion}

The recent JBDS guidance provides a clear and thorough approach to the safe and sensible use of an insulin sliding scale. This is especially important as several observational studies point to a strong association between hyperglycaemia and poor clinical
Figure 2. Infusion pump

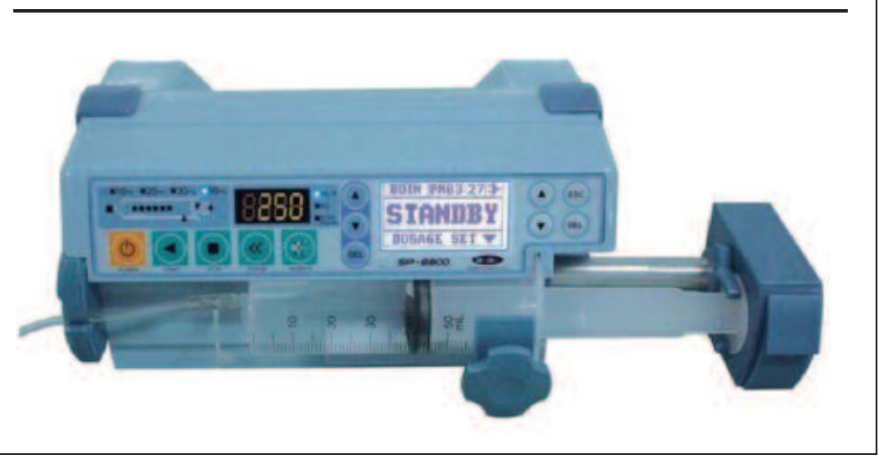

outcomes, including prolonged hospital stay, infection, disability after discharge from hospital and death. ${ }^{7-9}$ We were keen to assess our current hospital practice as part of projects to improve inpatient diabetes care in Brighton, ${ }^{10}$ and much of the JBDS guidance is a remarkable route to best practice (Figure 2).

It would appear that there is failure to achieve high rates of

Figure 3. Example of VRIII checklist

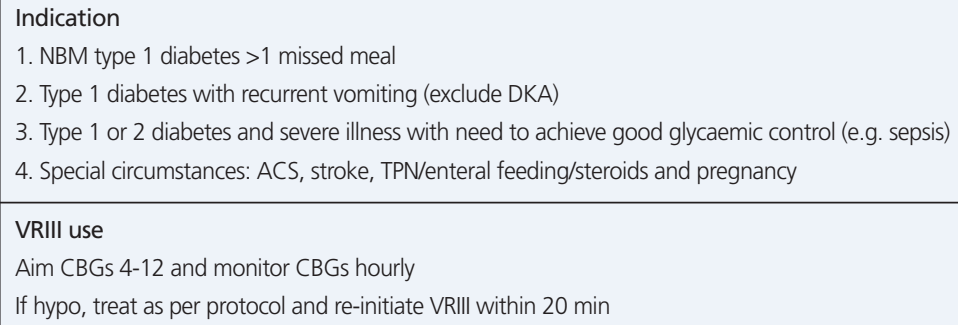

1. NBM type 1 diabetes $>1$ missed meal

2. Type 1 diabetes with recurrent vomiting (exclude DKA)

3. Type 1 or 2 diabetes and severe illness with need to achieve good glycaemic control (e.g. sepsis)

4. Special circumstances: ACS, stroke, TPN/enteral feeding/steroids and pregnancy

VRIII use

Aim CBGs 4-12 and monitor CBGs hourly

If hypo, treat as per protocol and re-initiate VRIII within 20 min

Assess fluid status

Check U\&Es

\begin{tabular}{l|ll}
\hline Prescribe VRIII and fluids as appropriate & Y/N & Y/N \\
\hline Omission of oral hypos/short acting and mix insulins & Y/N & Y/N \\
\hline Give long-acting insulin & Y/N & Y/N \\
\hline If persistent hypo/hyper, adjust the infusion regime & Y/N & Y/N \\
\hline Review the need for the VRIII & Y/N & Y/N
\end{tabular}

Comments

STOPPING THE VRIII

Ensure patient is able to eat and drink

Discontinue at meal time

For:

a. Insulin treated patients: Background (long-acting) insulin should have been continued. If not, this MUST be given prior to discontinuation of the VRIII. Give rapid-acting insulin with the meal and then stop the VRIII 30 min later

b. CSII (insulin pump) treated patients: Involve the diabetes team. Reconnect the CSII, start the normal basal rate insulin regimen, give a bolus dose of insulin with the meal and then stop the VRIII 30 min later

c. Non-insulin treated patients: Restart usual treatment. Ensure no contraindications to the previous hypoglycaemic therapy

Check CBG $1 \mathrm{~h}$ after discontinuing the VRIII and 4 hourly for the next $24 \mathrm{~h}$

\section{PLEASE CONTACT THE DIABETES TEAM}

1. If you are unable to achieve CBGs within target range

2. If your patient requires a VRIII for $>24 \mathrm{~h}$

3. If diabetes control was suboptimal prior to admission (i.e. recent pre admission $\mathrm{HbA} 1 \mathrm{c}>59 \mathrm{mmol} / \mathrm{mol}$ 
concordance on several of the dimensions relating to safe and effective VRIII usage when assessed in detail in this present audit. One major recurring factor appears to relate to the appropriateness of VRIII use in the context of dysglycaemia when another management strategy may be more appropriate. These, like other failings in its successful utilisation, most likely relate to insufficient knowledge and understanding. Similarly, the failure to continue basal insulin alongside VRIII can have an effect on outcomes, and both education and alterations to systems and processes can help overcome this.

It is apparent that overall practice in the clinical setting in relation to meeting the standards of VRIII usage is suboptimal within this small cohort of inpatients. Lessons need to be learnt and shared with the relevant inpatient teams, nurses, pharmacists and patients themselves. Whilst concordance is close to best practice in several areas, there are some specific problems that have been identified as being in need of improvement. When rolling out the new JBDS guidance, the aim is to deal with these in terms of changes to processes, but also via remedial education for all those involved in prescribing and administering, as 'trying harder' or 'more training' do not always work on their own in relation to multi-systems improvement. ${ }^{11}$

\section{Potential explanations for the above problems}

- General lack of understanding of the principles and pathophysiology of diabetes and insulin action amongst nursing and medical staff.

- Failure of clarity over the use and utility of VRIII.

- Traditional over-reliance on the inpatient diabetes nursing team to come and sort out diabetes problems.

- Lack of dedicated inpatient diabetes consultant sessions.

- Failure to make inpatient diabetes/glycaemic control management mandatory for junior doctors at trust induction.

- Failure to properly utilise the network linked glucose meter technology.

Our audit outcomes were therefore to prepare a protocol for the trust summarising the indications of VRIII, target CBGs, how and when to stop VRIII for patients with diabetes and to explore options for including this as part of both junior doctor and nursing training occurring at induction and on a ongoing statutory / mandatory basis.

Secondly, a prompt to prescribe basal insulin for patients with diabetes and when a referral should be made to the diabetes inpatient team, as well as an update to the current Trust-wide inpatient insulin prescribing and administration chart (VRIII section) needs to be considered. This may ultimately become more practical when electronic prescribing systems come into action in the 'near' future. Introducing a teaching session to all the professionals and raising awareness through a hospital-wide communication programme on the wards would also theoretically improve the results, ${ }^{12}$ as would the introduction of a dedicated 'Diabetes link' nurse in each of the major ward areas as part of a wider hospital diabetes improvement programme. The effect of such changes can be evaluated in future re-audits once the new guidance is implemented.

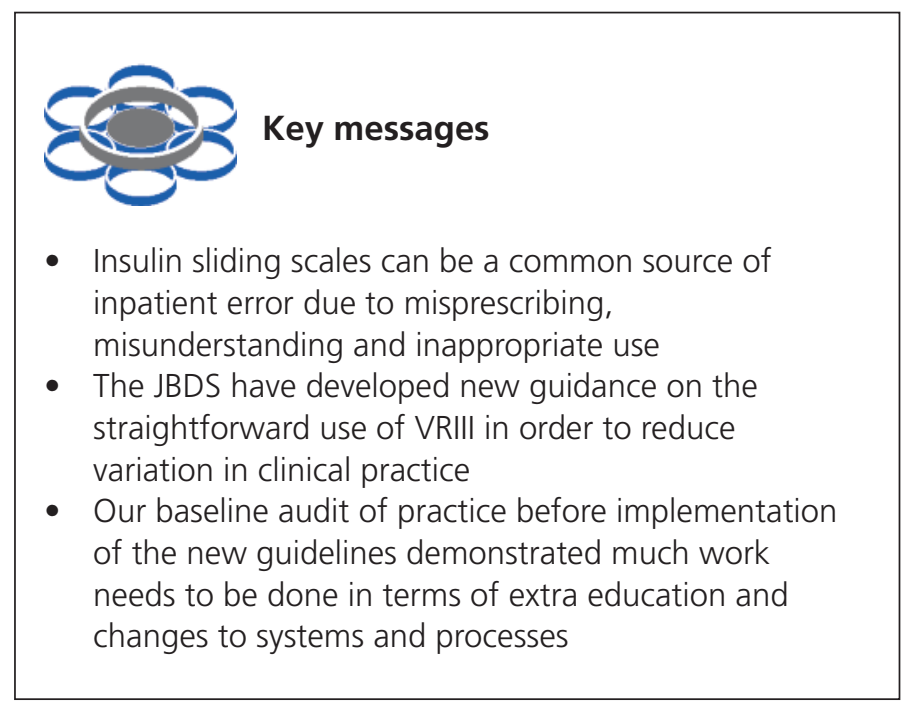

Criticisms of the JBDS guidance

It is difficult to find fault with the JBDS guidance itself, which is well thought out and comprehensive. Because of the problems identified in this audit and the fact that the majority of people with diabetes in the hospital setting are looked after by nondiabetologists, it would seem sensible - if not horribly reductionist - to produce and issue an even more abbreviated best practice guide for nurses, healthcare assistants and foundation doctors which can be accessed and referred to with ease, similar perhaps to the laminated cards used in many NHS hospital trusts which summarise microbiology guidance. An example of a comprehensive VRIII checklist is shown in Figure 3. Additionally, one may consider whether the necessity of a daily electrolyte check is essential in all cases (although the use of concurrent insulin and fluids necessary with a VRIII increases the risk of electrolyte disturbance). There should also be some reflection of patient factors to consider the individual at the centre of care and incorporate the concept of insulin self-administration and monitoring in parallel to develop a shared approach to inpatient diabetes management. This may necessitate the use of a joint monitoring document and a competency assessment to ensure safety and compliance (e.g., in the situation where basal insulin or mealtimes boluses continue to be given during VRIII usage).

\section{Conclusions}

The majority of patients on VRIII are managed by non-diabetic teams and practice may vary from set guidance due to unfamiliarity and lack of diabetes-specific knowledge. The appropriate use of VRIII needs to be tackled through remedial education, allied to improvements in systems and processes.

The JBDS standards themselves are good and relate to the real world. Refinements for future versions could include simplification for the non-specialist, removal of some non-essential processes and the inclusion of patient input/joint management for those who are able. It still remains the case that patients often know far more about their diabetes than the medical staff looking after them. 
Conflict of interest PG is the Editor of BJD and took no part in the handling or peer review of this manuscript following submission.

Acknowledgements Many thanks to Anna Potts, Andy Smith and the Diabetes Direct team.

\section{References}

1. Umpierrez G, Isaacs S, Bazargan N. Hyperglycemia: An independent marker of in-hospital mortality in patients with undiagnosed diabetes. $J$ Clin Endocrinol Metab 2002;87(3):978-82. http://dx.doi.org/10.1210/jc.2014-3415

2. Pickup JC, Keen H, Parsons JA, Alberti KG. Continuous subcutaneous insulin infusion: an approach to achieving normoglycaemia. Br Med J 1978;1(6107):204-07. http://dx.doi.org/10.1136/bmj.1.6107.204

3. Bruno A, Gregori D, Caropreso A. Normal glucose values are associated with a lower risk of mortality in hospitalized patients. Diabetes Care 2008;31(11):2209-10. http://dx.doi.org/10.2337/dc08-0672

4. Montori $\mathrm{V}$, Bistrian B, McMahon M. Hyperglycemia in acutely ill patients. JAMA 2002;288:2167-9. http://dx.doi.org/10.1001/jama.288.17.2167

5. National Diabetes Inpatient Audit. http://www.hscic.gov.uk/diabetesinpatientaudit (accessed 14 July 2015).

6. George S, Dale J, Stanisstreet D, et al. A guideline for the use of variable rate intravenous insulin infusion (VRIII) in medical inpatients. Br J Diabetes Vasc Dis 2015:15:82-5.

http://dx.doi.org/10.15277/bjdvd.2015.014

7. Cheung N, Wong V, McLean M. The Hyperglycemia: Intensive Insulin Infusion in Infarction (HI-5) study; a randomized controlled trial of insulin infusion therapy for myocardial infarction. Diabetes Care 2006;29(4): 765-70. http://dx.doi.org/10.2337/diacare.29.04.06.dc05-1894

8. Pomposelli J, Baxter J, Babineau T, et al. Early postoperative glucose control predicts nosocomial infection rate in diabetic patients. J Parenter Enteral Nutr 1998;22(2):77-81. http://dx.doi.org/10.1177/014860719802200277

9. Capes $S$, Hunt D, Malmberg K. Stress hyperglycemia and prognosis of stroke in non-diabetic and diabetic patients: a systematic overview. Stroke 2001;32(10):2426-32. http://dx.doi.org/10.1161/hs1001.096194

10. Grant P, Morel K, Lambert V, Burberry A, Vaughan N. Diabetes direct: solutions for inpatients with diabetes. Diabet Med 2015;32(Suppl 1):162-3.

11. Grant P. A multi-system approach to reducing insulin errors. Clinical Risk 2011;17(5):180-7. http://dx.doi.org/10.1258/cr.2011.011005

12. Grant $P$, Mustafa $O$, Malik R. Setting up an insulin safety group: a practical approach. Pract Diabetes 2012;29(4):160-2. http://dx.doi.org/10.1002/ pdi.1682

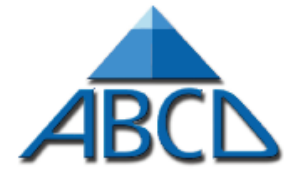

Associarion of British Clinical Diabetologists

\section{Dapagliflozin (Forxiga) Nationwide Audit in progress}

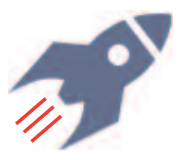

$A B C D$ launched a nationwide audit of dapagliflozin in the UK.

This audit is particularly important with dapaglifozin being the first of a new class of drugs for diabetes, the SGLT2 inhibitors. We have a chance to assess real clinical efficacy and safety of this new type of treatment by pooling our experience nationwide

\section{Does your centre use dapagliflozin (Forxiga)?}

If yes, REGISTER YOUR CENTRE! by contacting abcd.audits@diabetologists.org.uk

- you are able to analyse your local data easily

- you are invited to enter your patients' data into the online tool on N3 (the NHS secure network)

- the data will be automatically added to the national data in anonymised form

- we can provide easy-to-complete paper proformas for use in clinic if preferred

Please remember: - the more data, the more complete our understanding of this new treatment will be - all contributors will be listed in publications arising from data submission 